\title{
Portal vein thrombosis and Budd-Chiari syndrome as onset of polycythemia vera
}

\author{
Aurelio Seidita, ${ }^{1}$ Delia Sprini, ${ }^{1}$ Accursia Bongiovì, ${ }^{2}$ Tiziana Catalano, ${ }^{2}$ Filippo Barbiera, ${ }^{3}$ Maria Accardi, ${ }^{3}$ \\ Pasquale Mansueto, ${ }^{1}$ Antonio Carroccio ${ }^{2}$ \\ ${ }^{1}$ Internal Medicine, Department of Internal and Specialist Medicine, University Hospital of Palermo; ${ }^{2}$ Internal Medicine, Hospital \\ of Sciacca, ASP Agrigento; ${ }^{3}$ Radiology, Hospital of Sciacca, ASP Agrigento, Italy
}

\begin{abstract}
Budd-Chiari syndrome may be defined as a heterogeneous group of vascular disorders characterized by obstruction of hepatic venous return to the level of hepatic venules, supra-hepatic veins, inferior vena cava or right atrium. The main cause of this syndrome is represented by myeloproliferative diseases and, in particular, by polycythemia vera. The latter may cause multiple splanchnic thrombosis, including portal vein thrombosis, particularly important for its clinical outcomes (ascites, collateral vessels genesis, etc.). We report 2 cases of a Budd-Chiari syndrome induced by polycythemia vera characterized by an abnormal clinical onset, both as regards subjects' age (29 and 39 years old, respectively) and set of symptoms, signs and laboratory data. After a complete clinical, instrumental and genetic diagnosis, the patients were treated with combined therapy, using acetylsalicylic acid and hydroxyurea. The therapy proved successful and patients are still in follow up in our institution. Polycythemia vera should be suspected in patients affected with portal vein thrombosis and Budd-Chiari syndrome even if its clinical onset might be unusual. Every effort should be made to make a correct and early diagnosis in order to start appropriate therapy as soon as possible and to prevent patients from useless diagnostic and therapeutic treatments.
\end{abstract}

\section{Introduction}

Portal vein thrombosis (PVT) has a prevalence of $1 \%$ in the general population, and is mainly due to liver cirrhosis and myeloproliferative diseases. Among these, polycythemia vera (PV) and essential thrombocythemia (ET) represent at least $25 \%$ of possible causes of PVT. ${ }^{1,2}$ Moreover, myeloproliferative diseases are the most important cause of Budd-Chiari syndrome $(50 \%)$, as well as of all other splanchnic vein thrombosis (SVT). These are multifactorial diseases that might be caused by liver cirrhosis, neo-

Correspondence: Aurelio Seidita, Dipartimento di Medicina Interna e Specialistica (DIMIS), Via del Vespro 141, 90127 , Palermo, Italy.

Tel. +39.91.6552916 - Fax: +39.91.6552868.

E-mail: aurelio.seidita@unipa.it

Key words: Budd-Chiari syndrome, portal vein thrombosis, myeloproliferative disease, polycythemia vera.

Conflict of interest: the authors declare no potential conflict of interests.

This work is licensed under a Creative Commons Attribution NonCommercial 3.0 License (CC BY-NC 3.0).

CCopyright A. Seidita et al., 2013

Licensee PAGEPress, Italy

Italian Journal of Medicine 2013; 7:196-205

doi:10.4081/itjm.2013.196 plasms, abdominal infections, phlogosis and genetic or acquired abnormalities of coagulation pathway. ${ }^{2-4}$

We report 2 cases of young patients who were admitted to the Internal Medicine Department of the Giovanni Paolo II Hospital, Sciacca (AG), Italy, due to the sudden appearance of tense ascites and increase in transaminase which we found to be affected by BuddChiari syndrome as onset of an undiagnosed PV. We also made a brief revision of international literature to try to highlight the main etiopathogenetic and clinical aspects of the disease, and the latest indications for a correct therapeutic approach to these complex patients.

\section{Informed consent}

Informed consent was obtained from both patients for publication of their clinical cases.

\section{Case Report \#1}

A 29-year old Egyptian man was admitted to our Department due to sudden onset of abdominal pain and distension and diarrhea. Family and personal anamneses were uneventful as was his past medical history. Physical examination showed tense and globular abdomen, diffuse pain on superficial and deep palpation, abundant ascites and peripheral edema; no other signs or symptoms could be found. He, therefore, underwent routine hematochemical analysis that was normal except for microcytic hypochromic polycythemia, neutrophilic leukocytosis, hypoalbuminemia and transaminases, and 
increase in cholestasis indexes and inflammatory markers (Table 1). He underwent thorax and abdominal Xray (which proved normal) and abdomen ultrasound (US) scan which showed abundant ascites, liver with an non-homogeneous echogenicity pattern, increased portal vein diameter $(1.64 \mathrm{~cm})$ with few flow signs, and increased spleen volume (longitudinal diameter $15.7 \mathrm{~cm}$ ). This profile was compatible with portal vein thrombosis. For this, evacuative paracentesis was practised and $6 \mathrm{~L}$ of yellow citrine ascites were removed. Physical-chemical, microbiological and histological examination proved negative for exudates or neoplasms (Table 2). Broad-spectrum antibiotic therapy was started with cephalosporins plus fluoroquinolones, as well as antico- agulant therapy with low molecular weight heparin (LMWH) and warfarin. LMWH was administered till the international normalized ratio (INR) reached therapeutic values (range 2.5-3).

During the following days, despite therapy and albumin administration, due to the abovementioned hypoalbuminemia, rapid ascite formation recurred for which the patient underwent multiple evacuative paracentesis (always finding yellow citrine ascites). Moreover, due to persistence of diarrhea, macroscopic, physical-chemical, microbiological and parasitological examinations of the stool were performed. All proved negative so diarrhea was treated with symptomatic drugs (i.e. loperamide) with benefit.

Table 1. Abnormal hematochemical parameters in Case \#1.

\begin{tabular}{|c|c|c|c|}
\hline & Admittance & Discharge & Reference values \\
\hline Red blood cell count & $6.74 \times 10^{12} / \mathrm{L}$ & $5.84 \times 10^{12} / \mathrm{L}$ & $4.5-6 \times 10^{12} / \mathrm{L}$ \\
\hline Hemoglobin & $17.2 \mathrm{~g} / \mathrm{dL}$ & $16.8 \mathrm{~g} / \mathrm{dL}$ & $13-17 \mathrm{~g} / \mathrm{dL}$ \\
\hline Hematocrit & $53.9 \%$ & $52.9 \%$ & $42-54 \%$ \\
\hline Mean corpuscular volume & $74.2 \mathrm{fL}$ & $76.6 \mathrm{fL}$ & $78-95 \mathrm{fL}$ \\
\hline Mean corpuscular hemoglobin & $24.5 \mathrm{pg}$ & $24.9 \mathrm{pg}$ & $27.0-32.0 \mathrm{pg}$ \\
\hline AST & $59 \mathrm{U} / \mathrm{L}$ & $36 \mathrm{U} / \mathrm{L}$ & $10-40 \mathrm{U} / \mathrm{L}$ \\
\hline ALT & $69 \mathrm{U} / \mathrm{L}$ & $22 \mathrm{U} / \mathrm{L}$ & $9-41 \mathrm{U} / \mathrm{L}$ \\
\hline$\gamma-\mathrm{GT}$ & $121 \mathrm{U} / \mathrm{L}$ & $58 \mathrm{U} / \mathrm{L}$ & $8-61 \mathrm{U} / \mathrm{L}$ \\
\hline ALP & $315 \mathrm{U} / \mathrm{L}$ & $136 \mathrm{U} / \mathrm{L}$ & $80-270 \mathrm{U} / \mathrm{L}$ \\
\hline Total bilirubin & $2.47 \mathrm{mg} / \mathrm{dL}$ & $1.12 \mathrm{mg} / \mathrm{dL}$ & $0.20-1.60 \mathrm{mg} / \mathrm{dL}$ \\
\hline Albuminemia & $2.5 \mathrm{~g} / \mathrm{dL}$ & $3.5 \mathrm{~g} / \mathrm{dL}$ & $3.48-5.39 \mathrm{~g} / \mathrm{dL}$ \\
\hline CRP & $4.9 \mathrm{mg} / \mathrm{L}$ & $2.4 \mathrm{mg} / \mathrm{L}$ & $0-4 \mathrm{mg} / \mathrm{L}$ \\
\hline
\end{tabular}

AST, aspartate aminotransferase; ALT, alanine aminotransferase; $\gamma$-GT, gamma glutamyl transpeptidase; ALP, alkaline phosphatase; CRP, C-reactive protein.

Table 2. Ascites physical-chemical characteristics.

\begin{tabular}{lcc}
\hline & Case \#1 & Case \#2 \\
\hline Cell counts & & \\
White blood cells & $0.12 \times 10^{3} / \mu \mathrm{L}$ & $0.17 \times 10^{3} / \mu \mathrm{L}$ \\
Neutrophils & $0.07 \times 10^{3} / \mu \mathrm{L}$ & $0.07 \times 10^{3} / \mu \mathrm{L}$ \\
Lymphocytes & $0.01 \times 10^{3} / \mu \mathrm{L}$ & $0.02 \times 10^{3} / \mu \mathrm{L}$ \\
Monocytes & $0.01 \times 10^{3} / \mu \mathrm{L}$ & $<0.01 \times 10^{3} / \mu \mathrm{L}$ \\
\hline Chemical and physical examination & & \\
Color & Yellow & Yellow \\
Appearance & Limpid & Limpid \\
pH & 7.8 & 7.7 \\
Specific weight & 1010 & 1008 \\
Rivalta's test & Negative & Negative \\
Nitrites & Absent & Absent \\
Total protein & $12 \mathrm{~g} / \mathrm{L}$ & $20 \mathrm{~g} / \mathrm{L}$ \\
Microscopy & Negative & Negative \\
\hline Microbiology & No growth & No growth \\
\hline Cytology & Cytoatypia-free sediment & Cytoatypia-free sediment \\
& with some mesothelium cells & with some mesothelium cells and rare lymphocytes \\
\hline
\end{tabular}


Over the same days, glycated hemoglobin (HbA2) quantification and coagulation screening (both within normal limits), as well as d-dimer (significantly positive) and tumor marker evaluation ( $\alpha$-fetoprotein, CA 19.9, CA 15.3, and CEA, all negative) were performed, and all routine tests were repeated. There was a marked deterioration in liver and renal function, and microcytic hypochromic polycythemia and neutrophilic leukocytosis were confirmed. An abdominal computerized tomography (CT) scan with intravenous contrast was performed. This showed the presence of abundant ascites, an increase in liver volume characterized by non-homogeneous density, portal and splenic vein non-visualization, such as thrombosis, and splenomegaly, in absence of ischemic areas.

Some days later, the patient underwent another abdominal US scan which confirmed the abovementioned findings with, however, in addition the appearance of hepatic veins thrombosis (Budd-Chiari syndrome) and suggesting a hepatic profile compatible with liver stasis. This was confirmed by a further thorax and abdomen CT with intravenous contrast, which also showed the presence of small pleural effusion and splenic and portal vein partial recanalization. At the same time, we observed the contemporary and impressive improvement in liver function and cholestasis serological parameters, while red blood cell and white blood cell count abnormalities remained unchanged.

Meanwhile, according to a differential diagnosis of portal vein thrombosis, we evaluated blood markers of major and minor hepatotropic viruses, human immunodeficiency virus (HIV) types 1 and 2, and thrombophilic disorders (lupus anticoagulant, anti-cardiolipin antibodies, antinuclear antibodies HEp 2, native DNA antibodies, plasma anticoagulant protein $\mathrm{C}$, protein $\mathrm{S}$, and homocysteine). All were within normal range. Given his Egyptian origin, schistosomiasis was excluded by detection of serum anti-schistosome antibodies.

Due to the persistence of hypochromic microcytic polycythemia and portal vein thrombosis (verified by control abdominal US scan), we consulted a hematology specialist. After excluding presence of atypical lymphocyte subpopulations, the hematology specialist performed a bone marrow biopsy with evidence of an increase in erythroid and myeloid blast population. Therefore, according to international guidelines (Table 3), the patient underwent erythropoietin and mutation $J A K 2 \mathrm{~V} 617 \mathrm{~F}$ assays; both tested positive. We made a conclusive diagnosis of splenic, portal and hepatic vein thrombosis caused by PV hyperaggregability. We started combined therapy with oral acetylsalicylic acid $(100 \mathrm{mg} /$ day $)$ and oral hydroxyurea (15 mg bid). After the first three weeks of therapy, there was a marked reduction in red blood cells count, hemoglobin and hematocrit, up to a complete normalization at four weeks (Table 1). Furthermore, a consistent reduction in ascites was observed. Control thorax and abdominal CT with intravenous contrast was performed showing partial resolution of venous thrombosis (almost complete for the portal vein, to almost nothing for hepatic outflow veins). The patient continues periodic clinical, laboratory and imaging follow up at the hematology clinic of our Institution.

\section{Case Report \#2}

A 39-year old Sicilian woman was admitted to our Department due to sudden onset of abdominal distension and dyspnea. Family and personal anamneses were uneventful as was her past medical history with the exception of hypertension (father) and diabetes (grandmother). On physical examination, the abdomen appeared tense, globular, non-tender at superficial and deep palpation, with probable ascites that exceeded the transverse umbilical line of approximately $3 \mathrm{~cm}$. Examination of other organs and systems did not show anything relevant, excluding sinus tachycardia confirmed by electrocardiography. Routine blood tests showed pathological increase in transaminases, cholestasis and inflammation indexes. Moreover, the patient presented a clear microcytic normochromic polycythemia profile (Table 4). According to the abovementioned clinical and laboratory

Table 3. Proposed revised World Health Organization's criteria for polycythemia vera. Diagnosis requires the presence of both major criteria and 1 minor criterion or the presence of the first major criterion together with 2 minor criteria.

\section{Major criteria}

1. Hemoglobin $>18.5 \mathrm{~g} / \mathrm{dL}$ in men, $>16.5 \mathrm{~g} / \mathrm{dL}$ in women, or other evidence of increased red cell volume*

2. Presence of $J A K 2 \mathrm{~V} 617 \mathrm{~F}$ or other functionally similar mutation such as $J A K 2$ exon 12 mutation

\section{Minor criteria}

1. Bone marrow biopsy showing hypercellularity for age with trilineage growth (panmyelosis) with prominent erythroid, granulocytic, and megakaryocytic proliferation

2. Serum erythropoietin level below the reference range for normal

3. Endogenous erythroid colony formation in vitro

*Hemoglobin or hematocrit greater than $99^{\text {th }}$ percentile of method-specific reference range for age, gender, altitude of residence or hemoglobin greater than $17 \mathrm{~g} / \mathrm{dL}$ in men, $15 \mathrm{~g} / \mathrm{dL}$ in women if associated with a documented and sustained increase of at least $2 \mathrm{~g} / \mathrm{dL}$ from an individual's baseline value that can not be attributed to correction of iron deficiency, or elevated red cell mass greater than $25 \%$ above mean normal predicted value. Modified from Tefferi et al.,2007. ${ }^{5}$ 
findings, the patient underwent evacuative paracentesis, with removal of $4 \mathrm{~L}$ of flaxen serous fluid (physical-chemical, microbiological and histological examination proved negative for exudates or neoplasms) (Table 2). Abdominal CT (Figure 1) and magnetic resonance imaging (Figure 2 ) with intravenous contrast were, therefore, performed and these showed significant increase in liver volume with parenchymal non-homogeneous density, such as liver cirrhosis, hepatic veins, superior mesenteric vein, splenic vein and spleen-mesenteric confluence failure, showing as inveterate thrombosis, vicarages by various collateral

Table 4. Abnormal hematochemical parameters in Case \#2.

\begin{tabular}{|c|c|c|c|}
\hline & Admittance & Discharge & Reference values \\
\hline Red blood cell count & $7.46 \times 10^{12} / \mathrm{L}$ & $6.34 \times 10^{12} / \mathrm{L}$ & $4.5-6 \times 10^{12} / \mathrm{L}$ \\
\hline Hemoglobin & $16.5 \mathrm{~g} / \mathrm{dL}$ & $15.9 \mathrm{~g} / \mathrm{dL}$ & $13-17 \mathrm{~g} / \mathrm{dL}$ \\
\hline Hematocrit & $52.4 \%$ & $51.2 \%$ & $42-54 \%$ \\
\hline Mean corpuscular volume & $70.2 \mathrm{fL}$ & $75.8 \mathrm{fL}$ & $78-95 \mathrm{fL}$ \\
\hline Mean corpuscular hemoglobin & $22.1 \mathrm{pg}$ & $22.7 \mathrm{pg}$ & $27.0-32.0 \mathrm{pg}$ \\
\hline AST & $406 \mathrm{U} / \mathrm{L}$ & $50 \mathrm{U} / \mathrm{L}$ & $10-40 \mathrm{U} / \mathrm{L}$ \\
\hline ALT & $366 \mathrm{U} / \mathrm{L}$ & $38 \mathrm{U} / \mathrm{L}$ & $9-41 \mathrm{U} / \mathrm{L}$ \\
\hline$\gamma-\mathrm{GT}$ & $114 \mathrm{U} / \mathrm{L}$ & $42 \mathrm{U} / \mathrm{L}$ & $8-61 \mathrm{U} / \mathrm{L}$ \\
\hline ALP & $602 \mathrm{U} / \mathrm{L}$ & $127 \mathrm{U} / \mathrm{L}$ & $80-270 \mathrm{U} / \mathrm{L}$ \\
\hline Total bilirubin & $5.26 \mathrm{mg} / \mathrm{dL}$ & $1.24 \mathrm{mg} / \mathrm{dL}$ & $0.20-1.60 \mathrm{mg} / \mathrm{dL}$ \\
\hline Albuminemia & $3.0 \mathrm{~g} / \mathrm{dL}$ & $3.98 \mathrm{~g} / \mathrm{dL}$ & $3.48-5.39 \mathrm{~g} / \mathrm{dL}$ \\
\hline CRP & $4.6 \mathrm{mg} / \mathrm{L}$ & $1.0 \mathrm{mg} / \mathrm{L}$ & $0-4 \mathrm{mg} / \mathrm{L}$ \\
\hline
\end{tabular}

AST, aspartate aminotransferase; ALT, alanine aminotransferase; $\gamma$-GT, gamma glutamyl transpeptidase; ALP, alkaline phosphatase; CRP, C-reactive protein.

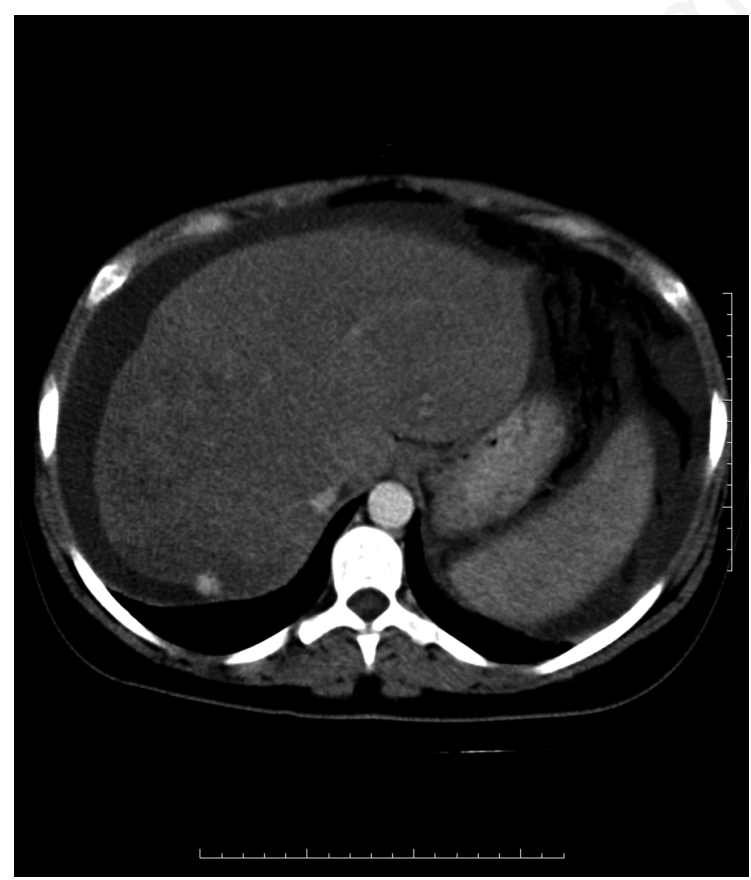

Figure 1. Computed tomography with intravenous contrast enhancement: increased liver volume, with heterogeneous density characterized by patchy enhancement, hepatic veins lack of opacification after intravenous administration of contrast, splenomegaly and abundant ascites.

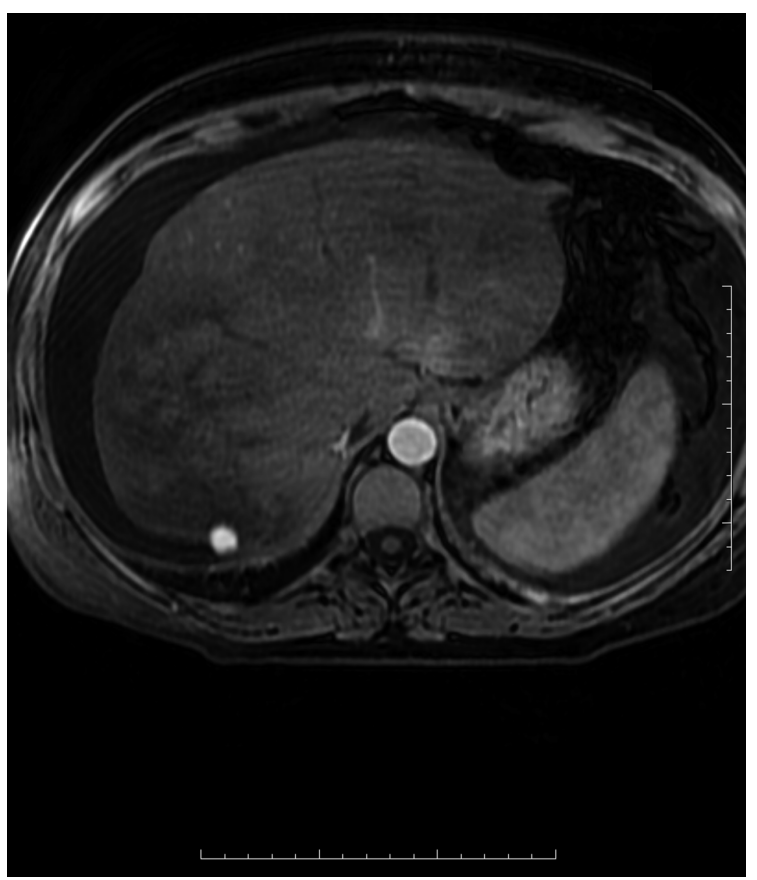

Figure 2. Magnetic resonance imaging with intravenous contrast enhancement: hepatomegaly with parenchymal inhomogeneous density, abundant ascites, mild splenomegaly, hepatic veins, superior mesenteric vein, splenic vein and spleen-mesenteric confluence failure shown. 
circulation, presence of probable regenerative nodules in S6 and S7, and a large amount of ascites. Furthermore, the patient underwent esophagogastroduodenoscopy that resulted normal except for a mild hyperemia of the gastric fundus, and echocardiography that was also normal.

Due to persistent polycythemia, persistence of massive ascites and evidence from imaging data, the patient underwent evaluation of blood markers of hepatotropic viruses, HIV types 1 and 2, and thrombophilic disorders (lupus anticoagulant, anti-cardiolipin antibodies, antinuclear antibodies HEp 2, native DNA antibodies, plasma anticoagulant protein $\mathrm{C}$, protein $\mathrm{S}$, and homocysteine) and of thyroid hormones. These were all within normal limits. We therefore started combined therapy with LMWH and oral anticoagulants until the target INR range (INR 2.5-3.5) was achieved.

Given the strong suspicion of polycythemia vera, genotyping for $J A K 2 \mathrm{~V} 617 \mathrm{~F}$ was tested and proved positive. In accordance with all the collected data, the patient was diagnosed with Budd-Chiari syndrome due to PV, and combined therapy with oral acetylsalicylic acid (100 mg/day) and oral hydroxyurea (15 mg bid) was started. The patient was discharged with a remarkable improvement in her clinical and laboratory condition (Table 4).

A subsequent abdominal CT control showed no change from the previous radiological profile, and the patient underwent transjugular intrahepatic portosystemic shunt (TIPS).

Currently the patient undergoes regular clinical and laboratory follow up in our Department, without further episodes of hepatic decompensation.

\section{Discussion}

Budd-Chiari syndrome can be defined as a heterogeneous group of disorders due to the obstruction, at any level (hepatic venules, large hepatic veins, inferior vena cava, right atrium), of the veins that guarantee liver blood outflow. ${ }^{6}$

It is an extremely rare disease the incidence of which is not well reported in literature, even if, recently, Rajani et al. have reported an incidence of approximately 1 case per 1 million inhabitants. It usually affects people between the fourth and fifth decade of life, and no difference according to gender has been reported. Mortality is highly variable, depending on the onset; almost $90 \%$ in fulminant forms, remaining, in the chronic forms, $55 \%$ at ten years from diagnosis. ${ }^{7}$

Pathogenesis may be attributable to a reduction in outflow from the liver leading to hepatic sinusoids and portal vein increased pressure, with a progressive stasis tendency and consequent hypoxic damage, as well as portal vein thrombosis. ${ }^{6,8} \mathrm{~A}$ further possible mechanism, related to blood stasis and hypoxic injury, which can promote hepatocellular damage, could be production of free radicals and the resulting oxidative damage. ${ }^{6,9}$

The disease culminates in centrilobular necrosis and, by well-known mechanisms of hepatic fibrosis and nodular regeneration, onset of terminal cirrhosis. ${ }^{6}$

Budd-Chiari syndrome etiology is not unique. It seems, in fact, that many factors, both acquired and genetic, may contribute to it. In particular, all hypercoagulable conditions play a major role, and can be identified in over $75 \%$ of all Budd-Chiari syndrome cases. ${ }^{10,11}$ Among these are myeloproliferative diseases. PV can be identified in $10-40 \%$ of all cases, while ET in a slightly lower rate. ${ }^{6,12-14}$ Even more evidence of the close relationship between Budd-Chiari syndrome and myeloproliferative diseases, an expansion of the endogenous erythroid-colony formation units in bone marrow, as to underlie a misunderstood myeloproliferative disease, can be observed in over $85 \%$ of patients in whom the etiology remains unknown (idiopathic). ${ }^{6,12,13}$ All other possible causes of Budd-Chiari syndrome and portal vein thrombosis, are listed in Table 5.

Clinical onset of Budd-Chiari syndrome is extremely variable and largely depends on the hepatic outflow vein occlusion speed. In fact, slow and progressive thrombosis ensures porto-systemic shunts genesis, with consequent minor stasis and hepatocellular damage. Vice versa it will result in a rapid occlusion, and massive or sub-massive liver damage might appear as fulminant and unexpected hepatic failure. ${ }^{11,15-17}$

Therefore, at least four forms of Budd-Chiari syndrome can be identified: fulminant, acute, subacute, and chronic. Patients with fulminant Budd-Chiari syndrome usually present with jaundice associated with an increase in liver function enzymes, nausea, vomiting, abdominal pain, and, within eight weeks of the onset, hepatic encephalopathy. In the acute form, there is a tendency to rapid and progressive liver parenchyma necrosis, with increase in transaminase, though smaller than that of the fulminant forms, in association with jaundice, pruritus, abdominal pain, and intractable ascites appearance. The subacute forms are certainly the most frequent, and their onset is insidious because ascite and laboratory abnormalities typical of hepatocellular damage are reduced, due to extensive collateral porto-systemic shunt formation. Finally, chronic Budd-Chiari syndrome is evident in patients with previous history of liver cirrhosis, as possible complication. Hepatomegaly is present in all four forms, while splenomegaly and esophageal varices are typical of subacute and chronic disease. ${ }^{6,15-17}$

Portal vein thrombosis and Budd-Chiari syndrome etiology can be considered to overlap, even if their clinical, laboratory and radiological profile might dif- 
fer. PVT usually involves the portal trunk extending, generally, toward the mesenteric vein and splenic vein. Its clinical onset may be acute or chronic. However, in approximately $35 \%$ of all cases it could be totally asymptomatic, only being confirmed after imaging. ${ }^{18}$

There is no agreement about the parameters needed to distinguish acute from chronic forms. As a general condition, a portal vein thrombosis whose symptoms developed no more than 60 days before hospitalization could be considered as acute. ${ }^{19,20}$ Acute thrombosis might be totally asymptomatic or characterized by unspecific bowel symptoms or signs (dyspepsia, abdominal colicky pain, nausea and vomiting, and mild abdominal distension) in most cases. However, severity of symptoms and signs strictly depends on type (complete or partial obstruction) and extension (portal, portal and splenic, portal splenic and mesenteric, etc.) of thrombosis. As a matter of fact, acute complete thrombosis extended to mesenteric vein could cause intestinal ischemia, bowel infarction and ileus. ${ }^{19-21}$

Amitrano et al. reported major bleeding from esophageal varices as possible acute PVT onset even if they point out this could be due more to underlying, still undiscovered cirrhosis rather than to acute onset of portal vein thrombosis. ${ }^{18}$

In acute PVT, an inflammatory systemic response is usually evidenced by acute inflammatory protein increase (e.g. fibrinogens, C-reactive protein, erythrocyte sedimentation rate). Liver function enzymes are within the normal range except for when an underlying liver disease may be evidenced; this could be due to an increase in arterial hepatic inflow to compensate for venous inflow reduction. ${ }^{21}$

Infective portal vein thrombosis is characterized by fever with chills, abdominal and liver pain, sudden onset of ascites, neutrophil leukocytosis, increase in inflammation markers and, sometimes, septic shock. In this framework, liver septic abscesses are common and blood cultures are usually positive for Bacteroides spp..$^{21}$

Chronic portal vein thrombosis, also known as portal cavernoma, is characterized by symptoms and signs connected with the collateral circles, which arise from the attempt to replace the portal circulation, creating links upstream and downstream of the stenosis, bypassing, generally, the hepatic filter. Location, size

Table 5. Budd-Chiari syndrome and portal vein thrombosis possible etiologies.

\section{Hypercoagulable states}

- Antiphospholipid syndrome

- Antithrombin deficiency

- Cancers

- Factor V Leiden mutation

- Methylenetetrahydrofolate reductase mutation TT677

- Myeloproliferative disorder

- Nephrotic syndrome

- Oral contraceptives

- Paroxysmal nocturnal hemoglobinuria

- Polycythemia rubra vera

- Pregnancy

- Prothrombin mutation G20210A

- Protein C deficiency

- Protein S deficiency

- Sickle cell disease

\section{Infections}

- Appendicitis

- Aspergillosis

- Cholangitis

- Cholecystitis

- Diverticulitis

- Liver abscess

- Schistosomiasis

- Umbilical vein infection
Complications of therapeutic interventions

- Alcohol injection

- Cholectomy

- Endoscopic sclerotherapy

- Fundoplication

- Gastric banding

- Hepatic chemoembolization

- Hepatobiliary surgery

- Islet cell injection

- Liver transplantation

- Peritoneal dialysis

- Radiofrequency ablation of hepatic tumor(s)

- Splenectomy

- TIPS procedure

- Umbilical vein catheterization
Inflammatory diseases

- Behçet's syndrome

- Inflammatory bowel disease

- Pancreatitis
Impaired portal vein flow

- Adrenal carcinoma

- Cirrhosis

- Cholangiocarcinoma

- Hepatocellular carcinoma

- Nodular regenerative hyperplasia

- Pancreatic carcinoma

- Renal cell carcinoma

- Sinusoidal obstruction syndrome

Others

- Bladder cancer

- Choledochal cyst

- Dacarbazine therapy

- Living at high altitude

- Trauma

TIPS, transjugular intrahepatic portosystemic shunt. Modified from Menon et al., 2004. ${ }^{4}$ 
and features of collateral circles vary: duodenal, antral, pancreatic and biliary veins could be involved.

To date, chronic portal vein thrombosis diagnosis has been mainly casual, due to ultrasonography studies often connected to other diseases or symptoms; so asymptomatic onset of this disease is rare. Sometimes jaundice, pruritus, fever, biliary pain, nausea and vomiting as well as cholangitis, cholecystitis and pancreatitis may signal onset of PVT. This condition is called portal cholangiopathy and its pathophysiological origin lies in the compression of the common bile duct by the collateral veins.

Variceal bleeding is not an unknown condition, whereas ascites and hepatic encephalopathy are rare. ${ }^{21,22}$ The first imaging approach to both Budd-Chiari syndrome and portal vein thrombosis is ultrasonography. In PVT, overdistension of portal vein could be found associated with the presence of hyperechoic materials inside of and disappearance of normal hepatic inflow. However, early thrombosis may appear as hypoechoic, causing a difficult detection, so a color Doppler imaging should be always performed as well to evaluate disappearance of normal hepatic flow. ${ }^{23,24}$

Ultrasonography in Budd-Chiari syndrome shows enlarged liver with caudate lobe hypertrophy. Hepatic veins could be partially or completely undetected. Otherwise, intraluminal hyperechoic material or dilatation of proximal tract may be observed. Inferior vena cava could be compressed by caudate lobe or show thrombus inside of it; rarely an aneurysmal dilatation can be detected. Excluding paraumbilical vein, also reported in portal vein thrombosis, appearance of intrahepatic collaterals is a specific sign of Budd-Chiari syndrome.

Color Doppler imaging can show no or abnormal direction flow in hepatic veins, whereas high-speed flow can be detected at the site of stenosis. Ascites may be detected easier in acute than in subacute or chronic Budd-Chiari syndrome. Both Budd-Chiari and portal vein thrombosis, in acute or chronic stage, have US measurable splenomegaly. ${ }^{24,25} \mathrm{CT}$ scans in portal vein thrombosis evidence well-defined intraluminal filling defect with central low attenuation, collateral circulation, engorgement of mesenteric veins, and mesenteric edema. Ultrasonography and CT are both specific and sensitive imaging techniques: the first is less invasive and provides a faster diagnosis and allows closer follow up; the second is more accurate and better identifies extension of thrombosis, especially towards problematic ultrasonography areas (mesenteric veins). ${ }^{18,21,24}$

The CT profile of acute Budd-Chiari syndrome is characterized by complete occlusion of hepatic veins (hypoattenuating) with severe ascites, with low peripheral and major central hepatic contrast enhancement. Splenomegaly is almost always evidenced, as are ascites. Subacute and chronic CT Budd-Chiari profiles are usually less clear. A nuanced hypoperfusion can be found in several hepatic parenchimal areas, together with collateral circulation and increase in diameter of the hepatic artery. The typical cirrhosis finding of regenerative nodules, with a diameter of $0.5-4 \mathrm{~cm}$, is usually evidenced by both contrasted and non-contrasted scans. ${ }^{24}$

In our Case \#1, early onset of portal vein thrombosis which appears to have preceded the occlusion of the hepatic veins, as well as what in our experience has shown to be higher frequency of other hepatocellular causes of injury delayed the diagnosis, requiring the patient to undergo a number of evacuative paracenteses and other invasive investigations, such as CT. We should also mention that only $4-7 \%$ of patients affected with polycythemia vera are under 40 years of age, and, of these, only $2-10 \%$ will develop a BuddChiari syndrome. ${ }^{26}$

Moreover, polycythemia vera is a far from common disease, occurring without differences of gender or race, especially in individuals older than 60 years. Annual incidence is highly variable, depending on the studies, ranging from $0.2 \times 10^{6}$ inhabitants/year in Japan to $28 \times 10^{6}$ inhabitants/year in Sweden. ${ }^{27-29}$ It is already known that myeloproliferative diseases are closely related to $J A K 2 \mathrm{~V} 617 \mathrm{~F}$ mutation, which can be identified in over $95 \%$ of polycythemia vera cases and over $60 \%$ of essential thrombocythemia and idiopathic myelofibrosis cases..$^{30,31}$ In addition, several recent studies pointed out that the presence of this mutation could be found in almost $35 \%$ of all splanchnic vein thrombosis forms, and in $41-59 \%$ of patients with Budd-Chiari syndrome. ${ }^{32,33}$ Association between $J A K 2$ mutation and the onset of splanchnic thrombosis appears to be linked to the presence of morphological and functional abnormalities of endothelial cells, leukocytes and platelets. In particular, some cellular receptors, such as CD11b, CD14, CD62P, CD63 and $\mathrm{P}$-selectin, seem to be over-expressed on the cytoplasmic membrane of these cells. Furthermore, P-selectin has an important role in attracting and activating leukocytes at the site of possible endothelial injury. $J A K 2$ mutation seems to be able to induce leukocyteplatelet aggregates and activate the coagulation cascade. ${ }^{34}$ Given this, the presence of other molecular microaggregates, including $\mathrm{CD} 11 \mathrm{~b} / \mathrm{CD} 62 \mathrm{P}$ and $\mathrm{CD} 11 \mathrm{~b} / \mathrm{CD} 42 \mathrm{~b}$, and of blood hyperviscosity, due to the high number of red blood cells, seem to explain the thrombophilic tendency of $J A K 2$ positive patients, beyond the presence or absence of a contemporary myeloproliferative disease. ${ }^{34-36}$ Moreover, an increased tissue factor synthesis by $J A K 2$ positive macrophages has been noticed in patients affected with SVT. So recently JAK2 V617F mutation has been considered among the possible diagnostic tools for the correct and 
early diagnosis of splanchnic vein thrombosis. ${ }^{34}$ Smalberg et al. recently demonstrated the importance of the $J A K 2$ mutations in splanchnic vein thrombosis. In 199 newly diagnosed patients, they noted that $46 / 1$ JAK2 haplotype expression (indicating the presence of single nucleotide polymorphism rs12343867) is significantly over-expressed in patients with $J A K 2 \mathrm{~V} 617 \mathrm{~F}$ mutation and splanchnic vein thrombosis compared to controls [heterozygous odds ratio (OR) 2.1, homozygous OR 4.1; $<<0.01]$. In patients who are $J A K \mathrm{~V} 617 \mathrm{~F}$ negative without myeloproliferative diseases and affected with splanchnic vein thrombosis, the haplotype 46/1 does not appear to cause increased risk of splanchnic vein thrombosis compared to controls (heterozygous OR 1.0 , homozygous $\mathrm{OR} 1.1 ; \mathrm{P}=0.98$ ). Finally, in the $J A K \mathrm{~V} 617 \mathrm{~F}$ negative patients with myeloproliferative diseases and splanchnic vein thrombosis, the risk of thrombosis seems significant compared to controls only in the case of homozygous mutation (heterozygous OR 0.6, homozygous OR 5.3; $\mathrm{P}=0.6$ ). The authors also emphasized that, in all JAK2 V617F mutation negative patients, haplotype 46/1 expression is, in any case, linked to an increased erythropoiesis. For these reasons they suggest adding the search for this haplotype to the diagnostic tools of splanchnic vein thrombosis diagnosis. ${ }^{31}$

A recent Italian study analyzed all the possible causes of death in 192 patients with polycythemia vera. The most important were found to be cardiovascular complicacy (36.4\%), including arterial thrombosis $(24 \%)$, venous thromboembolism $(5.2 \%)$ and other cardiovascular diseases $(7.2 \%)$. These are significantly higher than all other causes, including neoplasms $(30 \%)$, hemorrhages $(3.1 \%)$, polycythemia evolution $(2.6 \%$, spent phase $2.1 \%$, myelodysplasia $0.5 \%)$ and all other causes $(28.1 \%$, including hepatic failure $2.1 \%) .{ }^{37}$ The same Italian study evaluated the natural history of 1213 polycythemia vera treated patients, pointing out that the cumulative median survival exceeded 15 years, with overall mortality of 2.94 deaths/100 patients per year. ${ }^{40,41}$ Previous data evidenced a median survival of 18 months in untreated patients, thus confirming the importance of an early and correct treatment to drastically reduce complications, delay transformation into myeloid metaplasia with myelofibrosis or acute myeloid leukemia, and increase survival. $^{38}$

Polycythemia vera treatment is largely dependent on the age of the patient, risk of thrombosis and progressive development of thrombocytosis or splenomegaly, indexes of disease evolution..$^{38}$ The first therapeutic approach is still phlebotomy to maintain hematocrit consistently below $45 \%$. The only randomized study that compared phlebotomy with myelosuppressive therapy is the PVSG-01trial that stressed that the median survival in the study arm in which patients underwent only phlebotomy was significantly greater (13.9 years) than the arm undergoing therapy with agents such as radiophosphorus (11.8 years) and chlorambucil ( 8.3 years). This study, however, had great limitations. First of all, a large number of enrolled patients dropped out in the phlebotomy alone arm $(50 \%$ at 5 years and $90 \%$ at 10 years) due to lack of compliance, onset of complications or disease progression. ${ }^{38-41}$ So far, the approach based exclusively on phlebotomies is indicated only in patients at low risk of thrombosis (age $<60$ years and no history of previous thrombotic events).$^{38}$ In those patients in whom phlebotomy alone is not able to prevent complications, myelosuppressive therapy should be started as soon as possible. In subjects older than 70 years the use of radiophosphorus and busulfan or other alkylating agents is strongly advised, in combination with aspirin if the patient is at high thrombotic risk. Three randomized trials (PVSG01 trial, EORTC, FPSG) confirmed that these drugs significantly reduce thrombosis risk and delay myelofibrosis, indicating, also, that they should be used only in elderly patients because of their high mutagenicity. ${ }^{39,42-44}$ In all other patients (age $<70$ years), cytoreductive therapy with hydroxyurea should be considered. Many studies evaluated the efficacy and safety of this drug, compared both to untreated patients and those treated with alkylating agents, underlining the significant reduction in thrombosis risk $(5.6 \%$ vs $21.6 \%)$ and increased survival. ${ }^{38,45}$ Nevertheless, many doubts remain about the proven leukemogenicity and myelodysplastic potential of hydroxyurea. ${ }^{38,46}$

In younger patients (age $<60$ years), the Nordic MPD Group 2009 guidelines indicate using interferon $\alpha$ as first-line therapy. This would act by suppressing hematopoietic progenitor proliferation by a direct inhibitory effect on bone marrow progenitors and antagonizing platelet-derived growth factor and other cytokines, as transforming growth factor- $\beta$. Moreover, it seems not to have any teratogenic or leukemogenic effect. So far, however, there have been no randomized studies of its efficacy and safety. ${ }^{47-49}$

Regarding anagrelide, a novel platelet-lowering agent, the Nordic MPD Group guidelines reported its use only and exclusively in thrombosis related to cases of essential thrombocythemia. ${ }^{47,50,51}$ Antiplatelet therapy should be undertaken in all patients at high risk of thrombosis. Unfortunately, no blood chemistry parameter (platelet count, bleeding time, etc.) correlates with thrombotic risk in polycythemia vera patients. This explains why the beginning of antiplatelet therapy is entrusted exclusively to clinical judgment. The dose of aspirin (ASA) has been studied in several clinical trials, showing that, despite a minimum bleeding risk, administration of low-dose ASA is able to significantly reduce the risk of thrombosis..$^{52,53}$ 
In our Case \#1, we met with initial diagnostic and therapeutic difficulties due, mistakenly, to our previous experience given the nationality of the patient, the low incidence of this disease in young subjects and the abnormal presentation (portal vein thrombosis preceded the Budd-Chiari syndrome). Initially, to reduce ascites, we carried out several evacuative paracenteses simultaneously with anticoagulant therapy in an attempt to stabilize the patient's clinical condition and resolve the portal vein thrombosis. Later, when the occlusion of liver outflow veins was made explicit, contemporarily with exclusion of other Budd-Chiari syndrome etiologies, we looked for and identified the underlying myeloproliferative disease that was the basis of the disorder. To date, by continuous therapy with hydroxyurea and low-dose ASA, the patient is in good health and continues his clinical and laboratory follow up.

Case \#2 experienced a partial failure in therapy, for which reason the patient underwent TIPS. But given our previous experience with the young Egyptian patient, Case \#2 was significantly simpler, both as far as the diagnostic approach is concerned, and for patient clinical management. We succeeded in a short time to diagnose polycythemia vera and provide adequate therapy.

\section{Conclusions}

We report 2 cases of Budd-Chiari syndrome induced by a myeloproliferative disease (polycythemia vera), characterized by an abnormal clinical onset, both as regards subject's age (29 and 39 years old, respectively) and symptoms, signs and laboratory data (tense ascites due to portal vein thrombosis, and red blood cells and hematocrit values frankly not consistent with polycythemia vera, especially in Case \#1). Furthermore, we would like to stress the importance of Case \#1 for our professional development, which allowed us to achieve an early diagnosis of the disease in Case \#2.

\section{References}

1. Ogren M, Bergqvist D, Björck M, et al. Portal vein thrombosis: prevalence, patient characteristics and lifetime risk: a population study based on 23,796 consecutive autopsies. World J Gastroenterol 2006;12: 2115-9.

2. Denninger MH, Chaï $\mathrm{Y}$, Casadevall N, et al. Cause of portal or hepatic venous thrombosis in adults: the role of multiple concurrent factors. Hepatology 2000; 31:587-91.

3. Yoo EH, Jang JH, Park KJ, et al. Prevalence of overt myeloproliferative neoplasms and JAK2 V617F mutation in Korean patients with splanchnic vein thrombosis. Int J Lab Hematol 2011;33:471-6.

4. Menon KV, Shah V, Kamath PS. The Budd-Chiari syndrome. N Engl J Med 2004;350:578-85.
5. Tefferi A, Thiele J, Orazi A, et al. Proposals and rationale for revision of the World Health Organization diagnostic criteria for polycythemia vera, essential thrombocythemia, and primary myelofibrosis: recommendations from an ad hoc international expert panel. Blood 2007;110:1092-7.

6. Rajani R, Melin T, Björnsson E, et al. Budd-Chiari syndrome in Sweden: epidemiology, clinical characteristics and survival - an 18-year experience. Liver Int 2009;29:253-9.

7. Cazals-Hatem D, Vilgrain V, Genin P, et al. Arterial and portal circulation and parenchymal changes in Budd-Chiari syndrome: a study in 17 explanted livers. Hepatology 2003;37:510-9.

8. McCuskey RS. Morphological mechanisms for regulating blood flow through hepatic sinusoids. Liver 2000;20:3-7.

9. Valla D, Casadevall N, Lacombe C, et al. Primary myeloproliferative disorder and hepatic vein thrombosis: a prospective study of erythroid colony formation in vitro in 20 patients with Budd-Chiari syndrome. Ann Intern Med 1985;103:329-34.

10. Primignani M, Mannucci PM. The role of thrombophilia in splanchnic vein thrombosis. Semin Liver Dis 2008;28:293-301.

11. Yoo EH, Jang JH, Park KJ, et al. Prevalence of overt myeloproliferative neoplasms and JAK2 V617F mutation in Korean patients with splanchnic vein thrombosis. Int J Lab Hematol 2011;33:471-6.

12. Pagliuca A, Mufti GJ, Janossa-Tahernia M, et al. In vitro colony culture and chromosomal studies in hepatic and portal vein thrombosis - possible evidence of an occult myeloproliferative state. Q J Med 1990; 76:981-9.

13. De Stefano V, Za T, Ciminello A, et al. Causes of adult splanchnic vein thrombosis in the mediterranean area. Mediterr J Hematol Infect Dis 2011;3:e2011063.

14. Kocher G, Himmelmann A. Portal vein thrombosis (PVT): a study of 20 non-cirrhotic cases. Swiss Med Wkly 2005;135:372-6.

15. Buzas C, Sparchez Z, Cucuianu A, et al. Budd-Chiari syndrome secondary to polycythemia vera. A case report. J Gastrointestin Liver Dis 2009;18:363-6.

16. Iwasaki T, Kawai H, Oseki K, et al. Japanese case of Budd-Chiari syndrome due to hepatic vein thrombosis successfully treated with liver transplantation. Hepatol Res 2012;42:213-8.

17. Akoum R, Mahfoud D, Ghaoui A, et al. Budd-Chiari syndrome and heparin-induced thrombocytopenia in polycythemia vera: successful treatment with repeated TIPS and interferon alpha. J Cancer Res Ther 2009;5:305-8.

18. Amitrano L, Guardascione MA, Brancaccio V, et al. Risk factors and clinical presentation of portal vein thrombosis in patients with liver cirrhosis. J Hepatol 2004;40:736-41.

19. Chawla Y, Duseja A, Dhiman RK. Review article: the modern management of portal vein thrombosis. Aliment Pharmacol Ther 2009;30:881-94.

20. Malkowski P, Pawlak J, Michalowicz B, et al. Thrombolytic treatment of portal thrombosis. Hepatogastroenterology 2003;50:2098-100.

21. DeLeve LD, Valla DC, Garcia-Tsao G. American As- 
sociation for the Study Liver Diseases. Vascular disorders of the liver. Hepatology 2009;49:1729-64.

22. Condat B, Vilgrain V, Asselah T, et al. Portal cavernoma-associated cholangiopathy: a clinical and MR cholangiography coupled with MR portography imaging study. Hepatology 2003;37:1302-8.

23. Sacerdoti D, Serianni G, Gaiani S, et al. Thrombosis of the portal venous system. J Ultrasound 2007;10:12e21.

24. Brancatelli G, Vilgrain V, Federle MP, et al. BuddChiari syndrome: spectrum of imaging findings. AJR Am J Roentgenol 2007;188:W168-76.

25. Chaubal N, Dighe M, Hanchate V, et al. Sonography in Budd-Chiari syndrome. J Ultrasound Med 2006;25: 373-9.

26. Kutti J, Ridell B. Epidemiology of the myeloproliferative disorders: essential thrombocythaemia, polycythaemia vera and idiopathic myelofibrosis. Pathol Biol 2001;49:164-6.

27. Kurita S. Epidemiological studies of polycythemia vera in Japan. Nihon Ketsueki Gakkai Zasshi 1974;37:793-5.

28. McMullin MF, Bareford D, Campbell P, et al. General Haematology Task Force of the British Committee for Standards in Haematology. Guidelines for the diagnosis, investigation and management of polycythaemia/ erythrocytosis. Br J Haematol 2005; 130:174-95.

29. Tefferi A, Skoda R, Vardiman JW. Myeloproliferativeneoplasms: contemporary diagnosis using histology and genetics. Nat Rev Clin Oncol 2009;6:627-37.

30. Smalberg JH, Koehler E, Darwish Murad S, et al. European Network for Vascular Disorders of the Liver (EN-Vie). The JAK2 46/1 haplotype in Budd-Chiari syndrome and portal vein thrombosis. Blood 2011;117:3968-73.

31. Smalberg JH, Darwish Murad S, Braakman E, et al. Myeloproliferative disease in the pathogenesis and survival of Budd-Chiari syndrome. Haematologica 2006;91:1712-3.

32. Vladareanu AM, Popov V, Bumbea H, et al. Splanchnic vein thrombosis, the onset manifestation in JAK positive chronic myeloproliferative disorders neoplasms. J Med Life 2011;4:97-101.

33. Cervantes F, Arellano-Rodrigo E, Alvarez-Larrán A. Blood cell activation in myeloproliferative neoplasms. Haematologica 2009;94:1484-8.

34. Weber CA, Matzdorff AC, Gerriets T, et al. Circulating microemboli in patients with myeloproliferative disorders. Eur J Neurol 2007;14:199-205.

35. Falanga A, Marchetti M, Vignoli A, et al. Leukocyteplatelet interaction in patients with essential thrombocythemia and polycythemia vera. Exp Hematol 2005;33:523-30.

36. Gruppo Italiano Studio Policitemia (GISP). Polycythemia vera: the natural history of 1213 patients followed over 20 years. Ann Int Med 1995;123:656-64.

37. Pearson TC, Messinezy M, Westwood N, et al. A polycythemia vera updated: diagnosis, pathobiology, and treatment. Hematology Am Soc Hematol Educ Program 2000:51-68.

38. Berk PD, Goldberg JD, Donovan PB, et al. Therapeutic recommendations in polycythemia vera based on Polycythemia Vera Study Group protocols. Semin Hematol 1986;23:132-43.
39. Najean Y, Rain J-D. The very long term evolution of polycythemia vera: an analysis of 318 patients initially treated by phlebotomy or 32P between 1969 and 1981 . Semin Hematol 1997;34:6-16.

40. Thomas DJ, Marshall J, Russell RW, et al Effect of haematocrit on cerebral blood-flow in man. Lancet 1977;2:941-3.

41. Haanen C, Mathe G, Hayat M. Treatment of polycythaemia vera by radiophosphorus or busulphan: a randomized clinical trial. Br J Cancer 1981;44:75-80.

42. Najean Y, Rain JD. Treatment of polycythemia vera: use of 32P alone or in combination with maintenance therapy using hydroxyurea in 461 patients greater than 65 years of age. The French Polycythemia Study Group. Blood 1997;89:2319-27.

43. Brusamolino E, Salvaneschi L, Canevari A, Bernasconi C. Efficacy trial of pipobroman in polycythemia vera and incidence of acute leukemia. J Clin Oncol 1984;2:558-61.

44. Fruchtman SM, Mack K, Kaplan ME, et al. From efficacy to safety: a polycythemia vera study group report on hydroxyurea in patients with polycythemia vera. Semin Hematol 1997;34:17-23.

45. Sterkers Y, Preudhomme C, Laï JL, et al. Acute myeloid leukemia and myelodysplastic syndromes following essential thrombocythemia treated with hydroxyurea: high proportion of cases with $17 \mathrm{p}$ deletion. Blood 1998;91:616-22.

46. Nordic MPD Group. Guidelines for the diagnosis and treatment of patients with polycythemia vera, essential thrombocythemia and primary myelofibrosis; 2009. Available from: http://www.nordicmpd.org/files/ NMPD_guidelines_2009revision_pub.pdf

47. Elliott MA, Tefferi A. Interferon- $\alpha$ therapy in polycythemia vera and essential thrombocythemia. Semin Thromb Haemost 1997;23:463-72.

48. Talpaz M, Rakhit A, Rittweger K, et al. Phase I evaluation of a $40-\mathrm{kDa}$ branched-chain long-acting pegylated IFN-alpha-2a with and without cytarabine in patients with chronic myelogenous leukemia. Clin Cancer Res 2005;11:6247-55.

49. Petitt RM, Silverstein MN, Petrone M. Anagrelide for control of thrombocythemia in polycythemia and other myeloproliferative disorders. Semin Hematol 1997; 34:51-4.

50. Storen EC, Tefferi A. Long-term use of anagrelide in young patients with essential thrombocythemia. Blood 2001;97:863-6.

51. Tartaglia AP, Goldberg JD, Berk PD, Wasserman LR. Adverse effects of antiaggregating platelet therapy in the treatment of polycythemia vera. Semin Hematol 1986;23:172-6.

52. Gruppo Italiano Studio Policitemia (GISP). Low-dose aspirin in polycythemia vera. A pilot study. Br J Haematol 1997;97:453-6.

53. Di Nisio M, Barbui T, Di Gennaro L, et al. European Collaboration on Low-dose Aspirin in Polycythemia Vera (ECLAP) Investigators. The haematocrit and platelet target in polycythemia vera. Br J Haematol 2007;136:249-59. 